\title{
Principal Component Analysis based on Robust Estimators of the Covariance or Correlation Matrix: Influence Functions and Efficiencies
}

\author{
Christophe Croux * Gentiane Haesbroeck ${ }^{\dagger}$
}

\begin{abstract}
A robust principal component analysis can be easily performed by computing the eigenvalues and eigenvectors of a robust estimator of the covariance or correlation matrix. In this paper we derive the influence functions and the corresponding asymptotic variances for these robust estimators of eigenvalues and eigenvectors. The behavior of several of these estimators is investigated by a simulation study. Finally, the use of empirical influence functions is illustrated by a real data example.
\end{abstract}

Keywords: Influence Function, Principal Component Analysis, Robust Estimation, Robust Correlation Matrices.

AMS subject Classifications: $62 \mathrm{H} 25,62 \mathrm{~F} 35$

*ECARE and Institut de Statistique, Université Libre de Bruxelles, CP-139, Av. F.D. Roosevelt 50, B-1050 Brussels, Belgium, ccroux@ulb.ac.be.

†FEGSS, University of Liège, Bd du Rectorat 7, B-4000 Liège, Belgium, G.Haesbroeck@ulg.ac.be 


\section{Introduction}

One of the most popular techniques for analyzing multivariate data is principal component analysis (PCA). It consists of exploring the structure of a high-dimensional data set by projecting the observations onto the first principal components. These are obtained by computing the eigenvectors of the sample covariance or correlation matrix. The corresponding eigenvalues measure then the amount of information explained by the principal components. However, these estimators are extremely sensitive to outlying observations and conclusions drawn from contaminated principal components can be misleading. Several robustifications for PCA have been proposed (Jackson 1991, pages 365-371). Among these the replacement of the classical covariance or correlation matrix by a robust estimator is perhaps the most simple and intuitively appealing. Many simulation studies, starting with Devlin et al. (1981), have been carried out to find out which robust estimator should be used.

In this paper, a more formal comparison is undertaken by computing the influence functions for the estimators of the eigenvalues and eigenvectors. Corresponding asymptotic variances are also obtained. Results for M-estimators were already obtained by Jaupi and Saporta (1993), but our formulas are valid for any "regular" estimator, including high breakdown covariance matrix estimators. Several authors (Critchley 1985, Shi 1997) have suggested statistical diagnostics and graphical displays based on the influence function to detect influential points.

For every choice of the robust covariance matrix estimator, another robust PCA-method is obtained. An overview of existing estimators of multivariate location and scatter is given in Maronna and Yohai (1998). The words scatter matrix and covariance matrix will be abusively used as synonyms throughout this paper.

Three robust estimators $\left(t_{n}, \mathrm{C}_{n}\right)$ of multivariate location and scatter are considered in more detail: the M-estimator (Maronna 1976), the S-estimator (Rousseeuw and Leroy 1987, page 263, and Davies 1987) and the one-step reweighted Minimum Covariance Determinant (MCD) estimator (Rousseeuw 1985) which will be denoted by RMCD. Let us briefly review their definitions. Consider a sample of $p$-dimensional observations $x_{1}, \ldots, x_{n}$ and denote

$d\left(x_{i}, t, C\right)=\sqrt{\left(x_{i}-t\right)^{t} C^{-1}\left(x_{i}-t\right)}$ the statistical distance between $x_{i}$ and $t$, measured in the metric induced by the positive definite matrix $C$. 
M-estimates are implicitly defined by

$$
\begin{aligned}
t_{n} & =\frac{\sum_{i=1}^{n} w_{1}\left(d\left(x_{i}, t_{n}, \mathrm{C}_{n}\right)\right) x_{i}}{\sum_{i=1}^{n} w_{1}\left(d\left(x_{i}, t_{n}, \mathrm{C}_{n}\right)\right)} \\
\mathrm{C}_{n} & =\frac{1}{n} \sum_{i=1}^{n} w_{2}\left(d^{2}\left(x_{i}, t_{n}, \mathrm{C}_{n}\right)\right)\left(x_{i}-t_{n}\right)\left(x_{i}-t_{n}\right)^{t}
\end{aligned}
$$

where $w_{1}$ and $w_{2}$ are specified weight functions. Assuming monotonicity of $w_{2}$, Maronna (1976) showed that the M-estimation approach is less and less robust as the dimension increases since its breakdown point is at most $1 /(p+1)$.

Unlike the M-estimators, the S-estimators belong to the class of high breakdown estimators of multivariate location and scatter. They are defined as the solutions $\left(t_{n}, \mathrm{C}_{n}\right)$ to the problem of minimizing $\operatorname{det}(C)$ subject to

$$
\frac{1}{n} \sum_{i=1}^{n} \rho\left(d\left(x_{i}, t, C\right)\right)=b_{0}
$$

among all $(t, C) \in \mathbb{R}^{p} \times \operatorname{SPD}(p)$, with $\operatorname{SPD}(p)$ the set of all $p \times p$ symmetric and positive definite matrices. The constant $b_{0}$ is equal to $E_{F_{0}} \rho(\|x\|)$, with $F_{0}=\mathrm{N}_{p}(0, \mathbf{I})$.

Finally, the one-step reweighted MCD estimators are defined as

$$
\begin{aligned}
t_{n} & =\frac{\sum_{i=1}^{n} w_{i} x_{i}}{\sum_{i=1}^{n} w_{i}} \\
\mathrm{C}_{n} & =c_{1} \frac{\sum_{i=1}^{n} w_{i}\left(x_{i}-t_{n}\right)\left(x_{i}-t_{n}\right)^{t}}{\sum_{i=1}^{n} w_{i}}
\end{aligned}
$$

with $c_{1}=(1-\delta) / F_{\chi_{p+2}^{2}}\left(q_{\delta}\right)$ a consistency factor and $q_{\delta}=\chi_{p, 1-\delta}^{2}$ the upper $\delta$-percent point of a $\chi_{p}^{2}$ distribution. The weights are computed as

$$
w_{i}= \begin{cases}1 & \text { if } d^{2}\left(x_{i}, t_{n}^{0}, \mathrm{C}_{n}^{0}\right) \leq q_{\delta} \\ 0 & \text { otherwise }\end{cases}
$$

where $\left(t_{n}^{0}, \mathrm{C}_{n}^{0}\right)$ are the initial MCD estimates. For defining this MCD estimator, consider all the subsets of size $h(\leq n)$ from the sample and keep that subset whose covariance matrix has the smallest determinant. Then the location and scatter MCD estimates are given by the average and covariance matrix computed over this optimal subset. Typically, the size of the subset equals $h=[n(1-\alpha)]$, with $\alpha=0.5$ or $\alpha=0.25$. The breakdown points of MCD and RMCD are equal to $\alpha \%$. 
When numerical values or graphical displays are given, they correspond to the following choice of functions and constants. For the M-estimator, the weight functions are chosen according to Huber's proposal:

$$
w_{1}(y)=\frac{\psi_{H}\left(y, \sqrt{q_{\tau}}\right)}{y} \text { and } w_{2}(y)=\frac{\psi_{H}\left(y, q_{\tau}\right)}{\beta y} .
$$

where $\psi_{H}(y, k)=\max \{-k, \min \{y, k\}\}$ is Huber's psi function, $\beta$ is a constant making the scatter estimate Fisher consistent at normal models and $q_{\tau}=\chi_{p, 0.9}^{2}$. The function $\rho$ in the definition of the S-estimator is the Biweight function $\rho(y)=\min \left(\frac{y^{2}}{2}-\frac{y^{4}}{2 c_{0}^{2}}+\frac{y^{6}}{6 c_{0}^{4}}, \frac{c_{0}^{2}}{6}\right)$. To attain a breakdown point of $25 \%, c_{0}$ is implicitly defined by $\rho\left(c_{0}\right)=\frac{b_{0}}{r}$ with $r=0.25$. The breakdown point of the RMCD estimator will be the same as for the S-estimator, so $\alpha=0.25$, and the trimming parameter $\delta$ equals 0.025 as suggested by Rousseeuw and Van Driessen (1997).

The outline of the paper is as follows. Section 2 presents influence functions and asymptotic variances of eigenvalues and eigenvectors computed from a robust covariance matrix while Section 3 presents similar results for the correlation matrix. In Section 4, some simulations are conducted to compare the performance of the robust estimators introduced above to estimate the PCA eigenvalues and eigenvectors. As a byproduct, a comparison of the estimated correlation coefficients is also reported. In Section 5, the use of the influence function as a data analytic tool is illustrated. Section 6 contains some conclusions.

\section{Robust PCA based on the Covariance Matrix}

Let $x_{1}, \ldots, x_{n}$ be an i.i.d. sample drawn from a $p$-variate distribution $F$. Throughout the paper $F$ is assumed to be the normal distribution $\mathrm{N}(\mu, \Sigma)$ where $\mu \in \mathbb{R}^{p}$ and $\Sigma \in \operatorname{SPD}(p)$, the set of all $p \times p$ symmetric and positive definite matrices. Results can be easily shown to hold for any elliptically symmetric distribution $F$, but only for normal distributions one has that the principal components will be independent of each other (Hampel et al 1986, page 273). It is further supposed that $\Sigma$ has distinct eigenvalues $\lambda_{1}>\lambda_{2}>\ldots>\lambda_{p}>0$ with corresponding eigenvectors $v_{1}, v_{2}, \ldots, v_{p}$. The aim is to estimate these population eigenvalues and eigenvectors and to compute the corresponding influence functions. A generalization to multiple eigenvalues could be done as in Tanaka (1988). 
An influence function is essentially the first derivative of the functional version of an estimator. Let $\mathcal{F}$ denote the set of all distributions on $\mathbb{R}^{p}$ (or a very large subset of it). A map $\mathrm{C}: \mathcal{F} \rightarrow \operatorname{SPD}(p)$ which sends an arbitrary distribution $G \in \mathcal{F}$ to $\mathrm{C}(G)$ is a statistical functional corresponding to an estimator $\mathrm{C}_{n}$ of $\Sigma$ whenever $\mathrm{C}\left(F_{n}\right)=\mathrm{C}_{n}$, for every empirical distribution function $F_{n}$ associated with observations $x_{1}, \ldots, x_{n}$. For example, the statistical functional defined as

$$
\mathrm{C}(G)=E_{G}\left[\left(X-E_{G}[X]\right)\left(X-E_{G}[X]\right)^{t}\right]
$$

for any distribution $G$ having a second moment corresponds to the sample covariance matrix since

$$
\mathrm{C}\left(F_{n}\right)=\frac{1}{n} \sum_{i=1}^{n}\left(x_{i}-\bar{x}\right)\left(x_{i}-\bar{x}\right)^{t} .
$$

The notation $\mathrm{C}(X)$ instead of $\mathrm{C}(G)$ will be used whenever $X \sim G$. The functional representations of the eigenvectors and eigenvalues computed from $\mathrm{C}_{n}$ are denoted by ${ }^{{ }_{\mathrm{C}}, j}$ and $\lambda_{\mathrm{C}, j}$ for $j=1, \ldots, p$. Of course, $v_{\mathrm{C}, j}(G)$ and $\lambda_{\mathrm{C}, j}(G)$ are just the eigenvectors and eigenvalues of $\mathrm{C}(G)$, for every $G \in \mathcal{F}$. At the empirical distribution function, $v_{\mathrm{C}, j}\left(F_{n}\right)=$ $v_{\mathrm{C}_{n}, j}$ and $\lambda_{\mathrm{C}, j}\left(F_{n}\right)=\lambda_{\mathrm{C}_{n}, j}$. Throughout the paper, $\mathrm{C}$ is assumed to be Fisher consistent for $\Sigma$ at $F$, i.e. $\mathrm{C}(F)=\Sigma$, and affine equivariant, meaning that

$$
\mathrm{C}(A X+b)=A \mathrm{C}(X) A^{t}
$$

for any $b \in \mathbb{R}^{p}$ and any $p \times p$ non singular matrix $A$. This implies immediately that Fisher consistency also holds for the eigenvector and eigenvalue functionals,

$$
{ }^{v} \mathrm{C}, j_{j}(F)=v_{j} \text { and } \lambda_{\mathrm{C}, j}(F)=\lambda_{j} .
$$

The functionals $v_{\mathrm{C}, j}$ and $\lambda_{\mathrm{C}, j}$ are orthogonal equivariant in the sense that

$$
v_{\mathrm{C}, j}(\Gamma X)=\Gamma v_{\mathrm{C}, j}(X)
$$

and

$$
\lambda_{\mathrm{C}, j}(\Gamma X)=\lambda_{\mathrm{C}, j}(X)
$$

for $j=1, \ldots, p$ and for any $p \times p$ orthogonal matrix $\Gamma$.

To measure the robustness w.r.t. single outliers, it is common to compute their influence functions. By definition, the influence functions of $v_{\mathrm{C}, j}$ and $\lambda_{\mathrm{C}, j}$ are given by

$$
\mathrm{IF}\left(x, v_{\mathrm{C}, j} ; F\right)=\lim _{\varepsilon \downarrow 0} \frac{v_{\mathrm{C}, j}\left((1-\varepsilon) F+\varepsilon \Delta_{x}\right)-v_{\mathrm{C}, j}(F)}{\varepsilon}
$$


Table 1: Functions $\alpha_{\mathrm{C}}$ for the M, S, RMCD and classical estimator of the covariance matrix.

\begin{tabular}{|c|l|}
\hline $\mathrm{M}$ & $\alpha_{\mathrm{M}}(t)=\frac{p(p+2) w_{2}\left(t^{2}\right)}{p(p+2)+2 E_{F_{0}}\left[w_{2}^{\prime}\left(\|x\|^{2}\right)\|x\|^{4}\right]} \cdot$ \\
$\mathrm{S}$ & $\alpha_{\mathrm{S}}(t)=\frac{p \psi(t)}{\gamma_{1} t}$ where $\psi(t)=\rho^{\prime}(t)$ and $\gamma_{1}=\frac{E_{F_{0}}\left[\psi^{\prime}(\|y\|)\|y\|^{2}+(p+1) \psi(\|x\|)\|x\|\right]}{p+2}$. \\
$\mathrm{RMCD}$ & $\alpha_{\mathrm{RMCD}}(t)=\frac{d_{2}+2 d_{3}}{d_{2}} \alpha_{\mathrm{MCD}}(t)+\frac{1}{d_{2}} I\left(t \leq \sqrt{q_{\delta}}\right)$ where $q_{\delta}=\chi_{p, 1-\delta}^{2}, d_{2}=F_{\chi_{p+2}^{2}}\left(q_{\delta}\right)$, \\
& $d_{3}=-\frac{1}{2} F_{\chi_{p+4}^{2}}\left(q_{\delta}\right)$ and $\alpha_{\mathrm{MCD}}(t)=\frac{I\left(t \leq \sqrt{q_{\alpha}}\right)}{F_{\chi_{p+4}^{2}}\left(q_{\alpha}\right)}$ with $q_{\alpha}=\chi_{p, 1-\alpha}^{2}$. \\
$\operatorname{Cov}$ & $\alpha_{\mathrm{Cov}}(t)=1$
\end{tabular}

and

$$
\operatorname{IF}\left(x, \lambda_{\mathrm{C}, j} ; F\right)=\lim _{\varepsilon \downarrow 0} \frac{\lambda_{\mathrm{C}, j_{j}}\left((1-\varepsilon) F+\varepsilon \Delta_{x}\right)-\lambda_{\mathrm{C}, j}(F)}{\varepsilon}
$$

for $j=1, \ldots, p$. The Dirac measure $\Delta_{x}$ is the distribution putting all its mass on $x$. For more details on influence functions and statistical functionals, see Hampel et al (1986). When the influence function of the scatter estimator is known, the influence functions (2.1) and (2.2) can be easily derived, as will be shown in Theorem 1 below. Before that, we give a lemma characterizing the general form of the influence function of a scatter matrix estimator. All the proofs are kept for the Appendix.

Lemma 1. For any affine equivariant scatter matrix functional $\mathrm{C}$ possessing an influence function, there exist two functions $\alpha_{\mathrm{C}}, \beta_{\mathrm{C}}:[0, \infty[\rightarrow \mathbb{R}$ such that

$$
I F(x, \mathrm{C} ; F)=\alpha_{\mathrm{C}}(d(x))(x-\mu)(x-\mu)^{t}-\beta_{\mathrm{C}}(d(x)) \Sigma
$$

with $d^{2}(x)=(x-\mu)^{t} \Sigma^{-1}(x-\mu)$ and $F=N_{p}(\mu, \Sigma)$.

From now on, only robust scatter matrix estimators possessing an influence function will be considered. Among them, focus is put on the estimators M, S and RMCD as defined in Section 1. The influence functions of these scatter estimators have been derived by Huber (1981, page 226) for the M-estimator and by Lopuhaä (1989 and 1997) for S and for reweighted estimators. The influence function of RMCD depends on the influence function of the initial MCD estimator which can be found in Croux and Haesbroeck (1998). The 


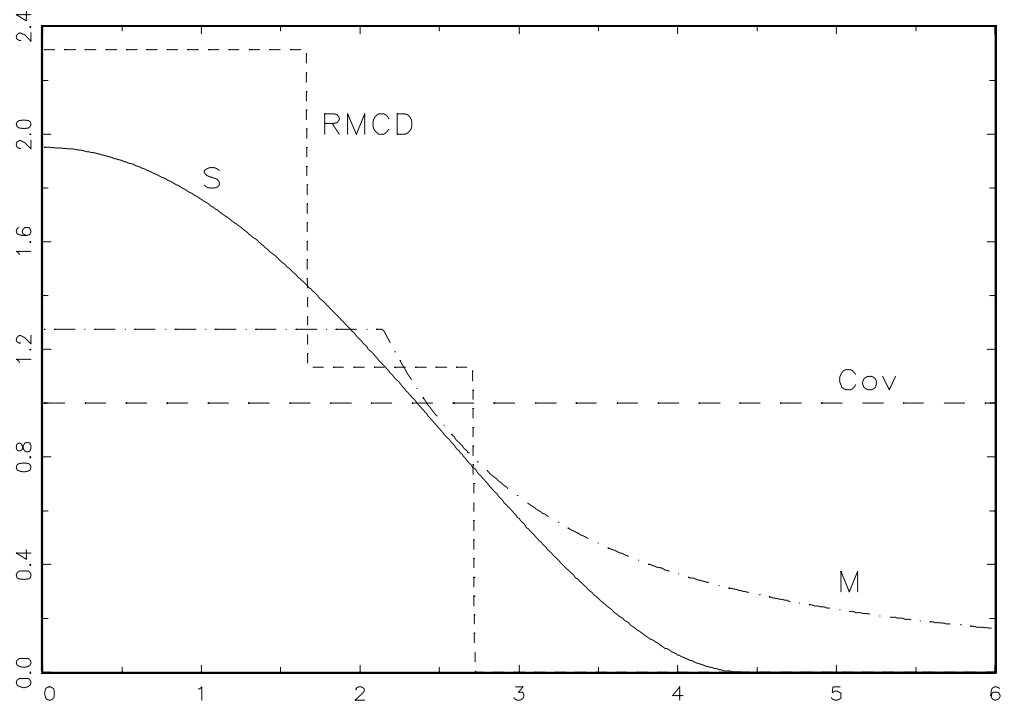

Figure 1. Examples of the function $\alpha_{\mathrm{C}}$ for some robust estimators.

corresponding functions $\alpha_{\mathrm{C}}$ are given in Table 1 and are plotted in Figure 1. The functions $\alpha_{\mathrm{S}}$ and $\alpha_{\mathrm{M}}$ are smooth while $\alpha_{\mathrm{RMCD}}$ is a step-function with two discontinuities: one at $\sqrt{q_{\alpha}}$ which is due to the initial estimator and the other one at $\sqrt{q_{\delta}}$ resulting from the weighting scheme. Both $\alpha_{\mathrm{RMCD}}(t)$ and $\alpha_{\mathrm{S}}(t)$ become zero after a certain rejection point while $\alpha_{\mathrm{M}}(t)$ only redescends to zero at infinity. All these functions are non increasing meaning that their contribution to the influence function decreases as the distance between $x$ and $\mu$ in the metric imposed by $\Sigma$ increases. The function $\alpha_{\mathrm{Cov}}$ is constant, implying that outliers are not given less weight.

Theorem 1. Let $F$ be a multivariate normal distribution with parameters $\mu$ and $\Sigma$. Define the scores of $x$ as $z_{k}=v_{k}^{t}(x-\mu)$ for $k=1, \ldots, p$ and let $d^{2}(x)=(x-\mu)^{t} \Sigma^{-1}(x-\mu)$. The influence functions of the eigenvectors and eigenvalues of $\mathrm{C}$ at $F$ are then given by

$$
\operatorname{IF}\left(x, \lambda_{\mathrm{C}, j} ; F\right)=\alpha_{\mathrm{C}}(d(x)) z_{j}^{2}-\beta_{\mathrm{C}}(d(x)) \lambda_{j}
$$

and

$$
I F\left(x, v_{\mathrm{C}, j} ; F\right)=\alpha_{\mathrm{C}}(d(x)) \sum_{\substack{k=1 \\ k \neq j}}^{p} \frac{z_{k} z_{j}}{\lambda_{j}-\lambda_{k}} v_{k}
$$

for $j=1, \ldots, p$. 
It follows now from Critchley (1985) that

$$
\mathrm{IF}\left(x, v_{\mathrm{C}, j} ; F\right)=\alpha_{\mathrm{C}}(d(x)) \operatorname{IF}\left(x, v_{\mathrm{Cov}, j} ; F\right) .
$$

The above equation confirms that the function $\alpha_{\mathrm{C}}$ needs to be interpreted as a downweighting function. A redescending $\alpha_{\mathrm{C}}$ function implies a bounded influence function for the eigenvectors.

In Figure 2, the influence function of the estimator $\lambda_{\mathrm{C}, 1}$ is plotted at $F=\mathrm{N}(0, \operatorname{diag}(2,1))$. Figure 3 represents the norm of the influence function of the first eigenvector $v_{\mathrm{C}, 1}$. For C, both the classical covariance matrix and the S-estimator have been considered. The curves obtained for the S-estimator resemble the curves obtained for the classical estimator at the center of the distribution. Points further away are downweighted by the robust estimator while they can still have a large influence on the usual covariance matrix. For the eigenvalues, the most influential points are along the direction of the corresponding eigenvector. The norm of the influence function of the eigenvector is the largest along the bisectors.

The influence function can also be helpful for computing asymptotic variances. If a functional $T$ corresponding to an estimator $T_{n}$ is "sufficiently regular", then

$$
\sqrt{n}\left(T_{n}-T(F)\right) \stackrel{d}{\rightarrow} \mathrm{N}_{p}(0, \operatorname{ASV}(T, F))
$$

with

$$
\operatorname{ASV}(T, F)=E_{F}\left[\operatorname{IF}(x, T ; F) \operatorname{IF}(x, T ; F)^{t}\right]
$$

(cfr. Hampel et al 1986, page 226). Taking (2.6) as definition of the asymptotic variance of a functional, the next corollary holds.

Corollary 1. With the notations of Theorem 1, one has

$$
\begin{aligned}
& A S V\left(\lambda_{\mathrm{C}, j}, F\right)=\lambda_{j}^{2} A S V\left(\mathrm{C}_{11}, F_{0}\right) \\
& A S V\left(v_{\mathrm{C}, j}, F\right)=A S V\left(\mathrm{C}_{12}, F_{0}\right) \sum_{\substack{k=1 \\
k \neq j}}^{p} \frac{\lambda_{k} \lambda_{j}}{\left(\lambda_{j}-\lambda_{k}\right)^{2}} v_{k} v_{k}^{t},
\end{aligned}
$$

for $j=1, \ldots, p$.

A formal proof of (2.5) for the robust eigenvectors and eigenvalues is beyond the scope of this paper. Boente (1987) provided regularity conditions in the case of M-estimators. Asymptotic 
(a)

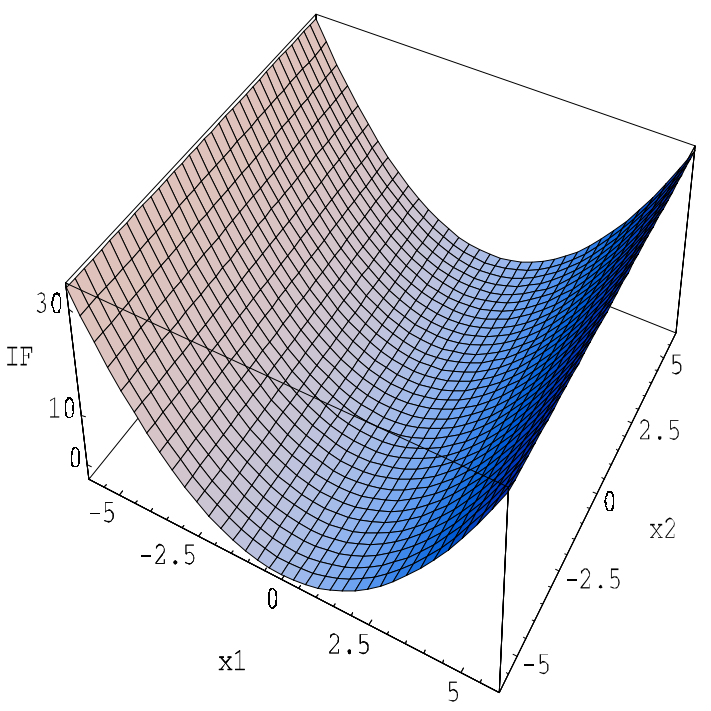

(b)

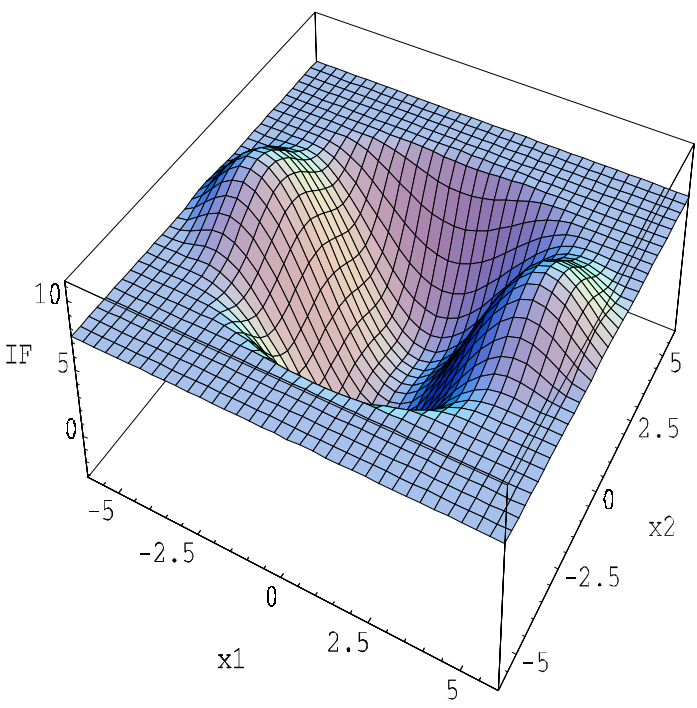

Figure 2. Influence function of the largest eigenvalue for (a) the classical covariance matrix and (b) the $S$-estimator at $F=N_{2}(0, \operatorname{diag}(2,1))$.

(a)

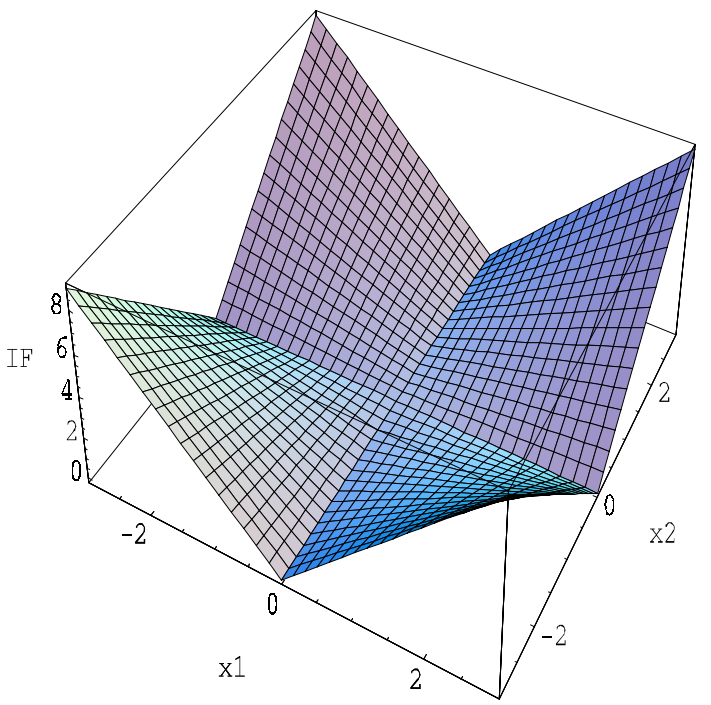

(b)

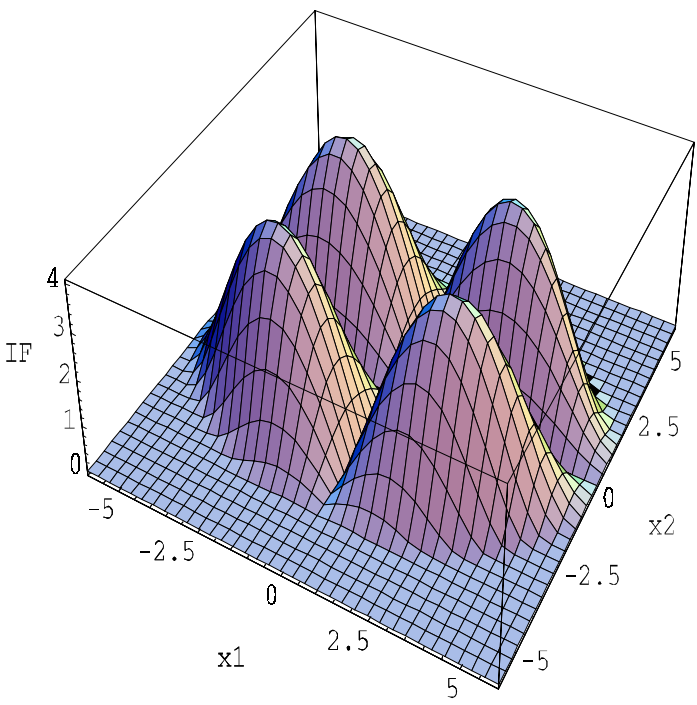

Figure 3. Norm of the influence function of the eigenvector corresponding to the largest eigenvalue for (a) the classical covariance matrix and (b) the $S$-estimator at $F=N_{2}(0, \operatorname{diag}(2,1))$. 
Table 2: Asymptotic efficiencies at the normal distribution for the eigenvalue and eigenvector estimators based on the RMCD, S and M scatter matrix estimators.

\begin{tabular}{|l|c|ccccc|}
\cline { 3 - 7 } \multicolumn{2}{c|}{} & $p=2$ & $p=3$ & $p=5$ & $p=10$ & $p=30$ \\
\hline \multirow{2}{*}{$\operatorname{Eff}\left(\lambda_{\mathrm{C}, j}, F_{0}\right)$} & $\mathrm{M}$ & 0.881 & 0.895 & 0.947 & 0.974 & 0.991 \\
& $\mathrm{~S}$ & 0.899 & 0.941 & 0.968 & 0.990 & 0.997 \\
& $\mathrm{RMCD}$ & 0.599 & 0.680 & 0.753 & 0.836 & 0.901 \\
\hline $\mathrm{Eff}\left(v_{\mathrm{C}, j}, F_{0}\right)$ & $\mathrm{M}$ & 0.920 & 0.947 & 0.969 & 0.986 & 0.996 \\
& $\mathrm{~S}$ & 0.850 & 0.924 & 0.967 & 0.988 & 0.997 \\
& RMCD & 0.635 & 0.742 & 0.820 & 0.873 & 0.933 \\
\hline
\end{tabular}

theory for eigenvectors and eigenvalues of the sample covariance matrix is given by Anderson (1963). Asymptotic efficiencies at normal distributions can be defined as

$$
\operatorname{Eff}\left(\lambda_{\mathrm{C}, j}, F\right)=\frac{\operatorname{ASV}\left(\lambda_{\mathrm{Cov}_{, j}}, F\right)}{\operatorname{ASV}\left(\lambda_{\mathrm{C}, j}, F\right)}=\frac{2}{\operatorname{ASV}\left(\mathrm{C}_{11}, F_{0}\right)}
$$

and

$$
\operatorname{Eff}\left(v_{\mathrm{C}, j}, F\right)=\left(\frac{\operatorname{det}\left(\operatorname { A S V } \left(v_{\left.\left.\mathrm{Cov}_{, j}, F\right)\right)}\right.\right.}{\operatorname{det}\left(\operatorname{ASV}\left(v_{\mathrm{C}, j}, F\right)\right)}\right)^{\frac{1}{p}}=\frac{1}{\operatorname{ASV}\left(\mathrm{C}_{12}, F_{0}\right)},
$$

since the maximum likelihood estimators of $\left(\lambda_{j}, v_{j}\right)$ at the normal model are given by the classical estimators (Joliffe 1986, page 41).

It is interesting to note that the efficiency of the eigenvalue estimators only depends on the efficiency of the diagonal elements of the scatter matrix estimator while the efficiency of the eigenvector estimators is computed from the efficiency of the off-diagonal elements. In Table 2, these efficiencies are reported for several values of $p$ and for the estimators of interest. The reweighted MCD estimator results in the lowest efficiency values, even if they are not too bad. The S and M-estimators are comparable w.r.t. their efficiency and should be preferred to the classical covariance matrix since the small loss of efficiency is compensated by a better robustness.

The asymptotic variances (2.7) and (2.8) are often used to construct large sample confidence intervals. For example, a large sample 100(1- $\alpha) \%$ confidence interval for $\lambda_{j}$ is 
provided by

$$
\left[\frac{\lambda_{\mathrm{C}_{n, j}}}{1+z_{\frac{\alpha}{2}} \sqrt{\frac{\mathrm{ASV}\left(\mathrm{C}_{11}, F_{0}\right)}{n-1}}}, \frac{\lambda_{\mathrm{C}_{n, j}}}{1-z_{\frac{\alpha}{2}} \sqrt{\frac{\mathrm{ASV}\left(\mathrm{C}_{11}, F_{0}\right)}{n-1}}}\right]
$$

where $z_{\frac{\alpha}{2}}$ is the upper $100\left(\frac{\alpha}{2}\right)$ th percentile of a standard normal distribution. For the covariance matrix, $\mathrm{ASV}\left(\mathrm{Cov}_{11}, F_{0}\right)=2$ and the usual formula is obtained again (cfr. Joliffe 1986, page 42$)$.

\section{Robust PCA based on the Correlation Matrix}

It is well known that the principal components are not independent of the scales in which the original variables are measured. It is therefore often recommended to derive the principal components from the correlation matrix. This Section deals with the influence functions of the eigenvalues and eigenvectors computed from that matrix.

For any matrix $B \in \mathbb{R}^{p \times p}$, let $\operatorname{diag}(B)$ denote the $p \times p$ diagonal matrix whose elements are the diagonal elements of $B$. The population version of the correlation matrix is defined as $P=\Sigma_{D}^{-\frac{1}{2}} \Sigma \Sigma_{D}^{-\frac{1}{2}}$ where $\Sigma_{D}=\operatorname{diag}(\Sigma)$. A natural estimator for $P$ is given by $\mathrm{R}_{n}=$ $\left(\mathrm{C}_{n}\right)_{D}^{-\frac{1}{2}} \mathrm{C}_{n}\left(\mathrm{C}_{n}\right)_{D}^{-\frac{1}{2}}$ with $\left(\mathrm{C}_{n}\right)_{D}=\operatorname{diag}\left(\mathrm{C}_{n}\right)$. The corresponding functional is defined as $\mathrm{R}(G)=$ $\mathrm{C}_{D}(G)^{-\frac{1}{2}} \mathrm{C}(G) \mathrm{C}_{D}^{-\frac{1}{2}}$, with $\mathrm{C}_{D}(G)=\operatorname{diag}(\mathrm{C}(G))$, for any distribution $G \in \mathcal{F}$. In this Section, $\lambda_{1}>\lambda_{2}>\ldots>\lambda_{p}>0$ denote the eigenvalues computed from $P$ with corresponding eigenvectors $v_{1}, v_{2}, \ldots, v_{p}$. The notations $\lambda_{\mathrm{R}, j}$ and $v_{\mathrm{R}, j}$ are obvious.

The following lemma proves that the influence function of the functional $\mathrm{R}$ can be easily worked out when the function $\alpha_{\mathrm{C}}$ appearing in the influence function of $\mathrm{C}$ is known. This lemma is then exploited to derive the influence function of the PCA functionals.

Lemma 2. For any $x \in \mathbb{R}^{p}$, denote $\tilde{x}=\Sigma_{D}^{-\frac{1}{2}}(x-\mu)$ its standardized version and $D_{\tilde{x}}=$ $\operatorname{diag}\left(\tilde{x} \tilde{x}^{t}\right)$. The influence function of the functional $\mathrm{R}$ can be written as

$$
I F(x, \mathrm{R} ; F)=\alpha_{\mathrm{C}}(d(x))\left\{\tilde{x} \tilde{x}^{t}-\left(\frac{D_{\tilde{x}} P+P D_{\tilde{x}}}{2}\right)\right\}
$$

where $d^{2}(x)=(x-\mu)^{t} \Sigma^{-1}(x-\mu), F=N_{p}(\mu, \Sigma)$ and $\alpha_{\mathrm{C}}$ is the real-valued function of (2.3).

The influence function of the correlation functional can be rewritten in the closer form

$$
\operatorname{IF}(x, \mathrm{R} ; F)=\alpha_{\mathrm{C}}(d(x)) \operatorname{IF}(x, \operatorname{Corr} ; F)
$$


where $\operatorname{IF}(x$, Corr; $F)$ is the influence function of the ordinary correlation matrix as derived in Devlin et al (1975). Only the function $\alpha_{\mathrm{C}}$ which already determined the form of $\operatorname{IF}(x, \mathrm{C} ; F)$ appears in $\operatorname{IF}(x, \mathrm{R} ; F)$. It may be easier to interpret the influence function when it is given element-wise. For the correlation functional, only the off-diagonal elements are of interest since $\operatorname{IF}\left(x, \mathrm{R}_{i i} ; F\right)=0$, as it should be. The element $(i, j)$ with $i \neq j$ is given by

$$
\mathrm{IF}\left(x, \mathrm{R}_{i j} ; F\right)=\alpha_{\mathrm{C}}(d(x))\left\{\tilde{x}_{i} \tilde{x}_{j}-P_{i j}\left(\frac{\tilde{x}_{i}^{2}+\tilde{x}_{j}^{2}}{2}\right)\right\}
$$

with $\tilde{x}_{i}=\left(x_{i}-\mu_{i}\right) / \sqrt{\Sigma_{i i}}(i=1, \ldots, p)$. Only the components $i$ and $j$ of $x$ influence $\operatorname{IF}\left(x, \operatorname{Corr}_{i j} ; F\right)$, but the other components may influence $d(x)$. When a robust estimator $\mathrm{C}$ is used, an extreme outlier in component $k$, which is however not outlying in two other components $i$ and $j$, may have zero influence on $\mathrm{R}_{i j}$. The influence functions of the correlation coefficients are of interest in their own right but are used here to derive the following Theorem.

Theorem 2. The influence function of the eigenvalues and eigenvectors of $\mathrm{R}$ at the model distribution $F=N(\mu, \Sigma)$ are given by

$$
\begin{aligned}
I F\left(x, \lambda_{\mathrm{R}, j} ; F\right) & =\alpha_{\mathrm{C}}(d(x))\left\{\tilde{z}_{j}^{2}-\lambda_{j} v_{j}^{t} D_{\tilde{x}} v_{j}\right\} \\
I F\left(x, v_{\mathrm{R}, j} ; F\right) & =\alpha_{\mathrm{C}}(d(x)) \sum_{\substack{k=1 \\
k \neq j}}^{p}\left(\tilde{z}_{k} \tilde{z}_{j}-\frac{\lambda_{k}+\lambda_{j}}{2} v_{j}^{t} D_{\tilde{x}} v_{k}\right) \frac{v_{k}}{\lambda_{j}-\lambda_{k}}
\end{aligned}
$$

where $\tilde{x}=\Sigma_{D}^{-\frac{1}{2}}(x-\mu), d^{2}(x)=(x-\mu)^{t} \Sigma^{-1}(x-\mu)$ and $\tilde{z}_{j}$ is the $j^{\text {th }}$ coordinate of the standardized $x$ in the basis of the eigenvectors, i.e. $\tilde{z}_{j}=v_{j}^{t} \tilde{x}$.

Once again, the function $\alpha_{\mathrm{C}}$ is responsible for the truncation of the influence functions derived for the usual correlation matrix since

$$
\operatorname{IF}\left(x, \lambda_{\mathrm{R}, j} ; F\right)=\alpha_{\mathrm{C}}(d(x)) \operatorname{IF}\left(x, \lambda_{\mathrm{Corr}, j} ; F\right)
$$

and

$$
\mathrm{IF}\left(x, v_{\mathrm{R}, j} ; F\right)=\alpha_{\mathrm{C}}(d(x)) \operatorname{IF}\left(x, v_{\mathrm{Corr}, j} ; F\right) .
$$

Two minor remarks complete this Section. Firstly, it follows from trace $(\mathrm{R})=p$ that $\sum_{j=1}^{p} \mathrm{IF}\left(x, \lambda_{\mathrm{R}, j} ; F\right)=0$. Secondly, the influence functions of the eigenvectors vanish for dimension $p=2$. 


\section{Finite-sample Experiment}

This Section uses simulations to compare the finite-sample performances of some robust estimators for estimating a correlation matrix and its principal components. The simulation set-up described in Devlin et al (1981) will be followed. The simulation consists of $m=1000$ replications of 6 -dimensional samples of $n=50$ observations generated from four different distributions. The robust estimators involved in this study are the $25 \%$ breakdown point one-step reweighted MCD estimator, the $25 \%$ breakdown Biweight S-estimator as well as the Huber M-estimator defined in Section 1. Note that the M-estimator was already included in Devlin et al's paper.

The population correlation matrix will be $P=\left(\begin{array}{cc}P_{1} & 0 \\ 0 & P_{2}\end{array}\right)$, with $P_{1}=\left(\begin{array}{ccc}1 & & \\ 0.95 & 1 & \\ 0.30 & 0.10 & 1\end{array}\right)$ and $P_{2}=\left(\begin{array}{ccc}1 & & \\ -0.499 & 1 & \\ -0.499 & -0.499 & 1\end{array}\right)$.

The values of interest are the elements of the correlation matrix $P$ and its eigenvalues $\left(\lambda_{1}=2.029, \lambda_{2}=\lambda_{3}=1.499, \lambda_{4}=0.943, \lambda_{5}=0.028, \lambda_{6}=0.002\right)$ and eigenvectors. The sampling distributions are taken as

1. The Normal distribution (NOR): $\mathrm{N}(0, P)$

2. A Symmetric Contaminated Normal (SCN) distribution: the mixture $0.9 \mathrm{~N}(0, P)+$ $0.1 \mathrm{~N}(0,9 P)$.

3. The multivariate Cauchy (CAU) which is defined as the distribution of $X=(\sqrt{S})^{-1} Y$, where $Y \sim \mathrm{N}(0, P)$ is independent of $S \sim \chi_{1}^{2}$.

4. An Asymmetric Contaminated Normal (ACN) distribution: the mixture 0.9 $\mathrm{N}(0, P)+$ $0.1 \mathrm{~N}(\mu, P)$, with (1) $\mu=0.537 \times v_{6}$ or $(2) \mu=50 \times v_{6}$ where $v_{6}$ is the eigenvector of $P$ corresponding to $\lambda_{6}$. The case $\mathrm{ACN}(1)$ corresponds to intermediate outliers while $\mathrm{ACN}(2)$ generates extreme outliers. It will appear that the $\mathrm{ACN}(1)$ contamination is not heavy enough to let the classical estimator break down. This is the reason why the $\mathrm{ACN}(2)$ configuration, not considered in the study of Devlin et al, was introduced. 
For computing the MCD estimator, the FAST-MCD algorithm of Rousseeuw and Van Driessen (1997) was applied, while the S-estimator was based on the SURREAL algorithm of Ruppert (1992). The iterative procedure given in Devlin et al (1981) for computing the M-estimator was used. All algorithms were implemented in GAUSS.

To assess the performance of the estimators of the elements of $P$, the finite-sample bias was computed as well as the Mean Squared Error. As in Devlin et al's study, the reported MSE's are defined as

$$
\operatorname{MSE}\left(\rho_{i j}\right)=\frac{1}{m} \sum_{k=1}^{m}\left(\hat{\rho}_{i j}^{(k)}-\rho_{i j}\right)^{2}
$$

where $\rho_{i j}=\frac{1}{2} \ln \left(\frac{1+P_{i j}}{1-P_{i j}}\right)$ is the Fisher's $z$ transform of the correlation coefficient $P_{i j}$ and $\hat{\rho}_{i j}^{(k)}$ its estimate computed from the $k$ th generated sample.

The biases of the different estimators are not reported since they were comparable across all sampling distributions (except for the M-estimator at the ACN-distributions, where the biases for the smaller correlations were higher). At the uncontaminated normal the classical correlation estimator is of course the most efficient, but the loss for the M and S-estimators is almost negligible. It can be seen that the Huber M-estimator outperforms the classical correlation at most other schemes. This explains why Devlin et al recommended the Huber M-estimator. At the $\mathrm{ACN}(2)$ configuration, one sees however that the M-estimator breaks down, confirming its lower breakdown point. This is not the case for the S-estimator, which appears to be the most robust at the asymmetric contamination distributions. Moreover, also for the other sampling schemes the S-estimator yields MSEs comparable to the M-estimator. The only exception is the Cauchy distribution, where the M-estimator behaves extremely well. The other competitor, RMCD, is clearly less efficient than the S-estimator, even at the contaminated distributions. As a first conclusion, one may say that the S-estimator seems to be the best estimator for the correlation coefficients.

The precision of the estimators for the eigenvalues of $P$ was measured by

$$
\operatorname{MSE}\left(\ln \lambda_{i}\right)=\frac{1}{m} \sum_{k=1}^{m}\left(\ln \hat{\lambda}_{i}^{(k)}-\ln \lambda_{i}\right)^{2},
$$

where $\hat{\lambda}_{i}^{(k)}$ is the estimate for the $i$ th eigenvalue computed from the $k$ th generated sample. Not very surprisingly, the same observations as for the correlation coefficients can be made from Table 3. The S-estimator turns out to be preferable, thanks to its relatively high 
Table 3: $1,000 \times$ MSE of the $z$-transforms of the estimators of the elements of the correlation matrix and $100 \times \mathrm{MSE}$ of the estimators of the logs of the eigenvalues under five different sampling schemes. The classical estimator is indicated by Corr, the Huber M-estimator by $\mathrm{M}$, and the results based on the reweighted MCD and the S-estimator are in the two other columns.

\begin{tabular}{|c|c|cccc|c|cccc|}
\hline dist & $1,000 \times \mathrm{R}_{i j}$ & \multicolumn{3}{|c|}{$1,000 \times \mathrm{MSE}\left(\tilde{\rho}_{i j}\right)$} & $\lambda_{i}$ & \multicolumn{4}{|c|}{$100 \times \mathrm{MSE}\left(\ln \lambda_{i}\right)$} \\
& & $\mathrm{Corr}$ & $\mathrm{M}$ & $\mathrm{S}$ & $\mathrm{RMCD}$ & & $\mathrm{Corr}$ & $\mathrm{M}$ & $\mathrm{S}$ & $\mathrm{RMCD}$ \\
\hline & 950 & 21 & 21 & 23 & 39 & 2.029 & 1 & 1 & 1 & 3 \\
$\mathrm{~N}$ & 300 & 21 & 22 & 24 & 39 & 1.499 & 1 & 1 & 1 & 2 \\
$\mathrm{O}$ & 100 & 20 & 22 & 24 & 38 & 1.499 & 3 & 3 & 3 & 5 \\
$\mathrm{R}$ & -499 & 22 & 23 & 21 & 40 & 0.943 & 3 & 3 & 4 & 12 \\
& -499 & 24 & 25 & 23 & 43 & 0.028 & 8 & 9 & 10 & 20 \\
& -499 & 21 & 22 & 22 & 39 & 0.002 & 8 & 8 & 8 & 22 \\
\hline & 950 & 54 & 24 & 28 & 38 & 2.029 & 4 & 1 & 2 & 2 \\
$\mathrm{~S}$ & 300 & 54 & 24 & 28 & 38 & 1.499 & 2 & 1 & 1 & 1 \\
$\mathrm{C}$ & 100 & 55 & 24 & 28 & 37 & 1.499 & 8 & 3 & 4 & 5 \\
$\mathrm{~N}$ & -499 & 56 & 26 & 27 & 37 & 0.943 & 13 & 4 & 5 & 10 \\
& -499 & 55 & 27 & 29 & 36 & 0.028 & 23 & 10 & 13 & 17 \\
& -499 & 57 & 25 & 27 & 35 & 0.002 & 22 & 9 & 11 & 19 \\
\hline & 950 & 1128 & 34 & 63 & 71 & 2.029 & 43 & 2 & 5 & 5 \\
$\mathrm{C}$ & 300 & 1428 & 38 & 71 & 72 & 1.499 & 130 & 1 & 2 & 2 \\
$\mathrm{~A}$ & 100 & 1400 & 38 & 71 & 70 & 1.499 & 481 & 5 & 9 & 10 \\
$\mathrm{U}$ & -499 & 1400 & 35 & 65 & 69 & 0.943 & 877 & 8 & 19 & 22 \\
& -499 & 1314 & 35 & 61 & 68 & 0.028 & 764 & 14 & 30 & 33 \\
& -499 & 1361 & 35 & 62 & 67 & 0.002 & 1042 & 15 & 31 & 37 \\
\hline & 950 & 21 & 22 & 25 & 36 & 2.029 & 1 & 1 & 1 & 2 \\
$\mathrm{~A}$ & 300 & 21 & 22 & 22 & 38 & 1.499 & 1 & 1 & 1 & 1 \\
$\mathrm{C}$ & 100 & 20 & 22 & 23 & 38 & 1.499 & 3 & 3 & 3 & 5 \\
$\mathrm{~N}$ & -499 & 23 & 23 & 22 & 36 & 0.943 & 3 & 4 & 4 & 10 \\
$(1)$ & -499 & 23 & 24 & 23 & 33 & 0.028 & 5 & 6 & 7 & 17 \\
& -499 & 21 & 22 & 22 & 36 & 0.002 & 567 & 458 & 381 & 18 \\
\hline & 950 & 21 & 23 & 25 & 35 & 2.029 & 18 & 15 & 1 & 2 \\
$\mathrm{~A}$ & 300 & 21 & 23 & 25 & 41 & 1.499 & 7 & 8 & 1 & 1 \\
$\mathrm{C}$ & 100 & 20 & 22 & 25 & 41 & 1.499 & 28 & 27 & 3 & 5 \\
$\mathrm{~N}$ & -499 & 8366 & 6170 & 24 & 36 & 0.943 & 1188 & 863 & 4 & 10 \\
$(2)$ & -499 & 8331 & 6139 & 23 & 34 & 0.028 & 17 & 13 & 11 & 17 \\
& -499 & 8345 & 6150 & 24 & 31 & 0.002 & 368 & 585 & 9 & 19 \\
\hline
\end{tabular}


efficiency combined with good robustness properties. There is one case where the S-estimator breaks down while RMCD does not: $\lambda_{6}$ for $\operatorname{ACN}(2)$. Apparently, the discontinuous character of RMCD (cfr. Figure 1) may lead to more robust solutions in some cases, at the price of a loss of efficiency.

It is also of interest to compare the estimators w.r.t. their performance to estimate the eigenvectors of the correlation matrix $P$. The estimations should be close to the true vector, i.e. the vectors $v_{j}$ and $v_{\mathrm{C}_{n}, j}$ should be collinear. To measure their closeness, the cosine of the angle $\hat{\theta}_{j}$ they form is used. (For $j=2$ and $3, \hat{\theta}_{j}$ should be taken as the angle between $v_{\mathrm{C}_{n}, j}$ and its projection onto the space spanned by $v_{2}$ and $v_{3}$ since $\lambda_{2}=\lambda_{3}$.) Figure 4 gives the empirical cumulative distribution functions of the realizations of $\left|\cos \hat{\theta}_{j}\right|$ over the $m=1000$ replications under the distributions NOR and ACN for the M and S-estimators. Similar figures were given in Devlin et al (1981), but only at the NOR distribution. The

distributions of $\left|\cos \hat{\theta}_{5}\right|,\left|\cos \hat{\theta}_{3}\right|$ and $\left|\cos \hat{\theta}_{2}\right|$ are omitted since the first one behaves like $\left|\cos \hat{\theta}_{6}\right|$, and the two others like $\left|\cos \hat{\theta}_{4}\right|$.

Comparing the two columns of Figure 4, one sees that the S-estimator is more robust than the M-estimator. Indeed, since the values of $\left|\cos \hat{\theta}_{j}\right|$ should be close to one, the cumulative distribution function should be peaked towards one. This is no longer true for the Mestimator at the $\mathrm{ACN}(2)$ distribution, while the S-estimator still finds good estimates for the eigenvectors in that case. The same exercise has been done for the RMCD estimator, yielding results which are even slightly better than for the S-estimator. Therefore, the RMCD estimator can still play its role in an exploratory analysis, when efficiency and inference issues are less important.

\section{The Empirical Influence Function}

Several authors (Critchley 1985, Shi 1997) have proposed local influence measures to detect influential points in a principal component analysis. Their measures were based, however, on the non-robust sample covariance matrix. Jaupi and Saporta (1993) introduced empirical influence measures based on M-estimators. In this Section the same approach is followed, now using a high breakdown estimator of multivariate scatter. If $t_{n}$ and $\mathrm{C}_{n}$ are estimates obtained from the sample $x_{1}, \ldots, x_{n}$, then the empirical influence functions (EIF) for the 

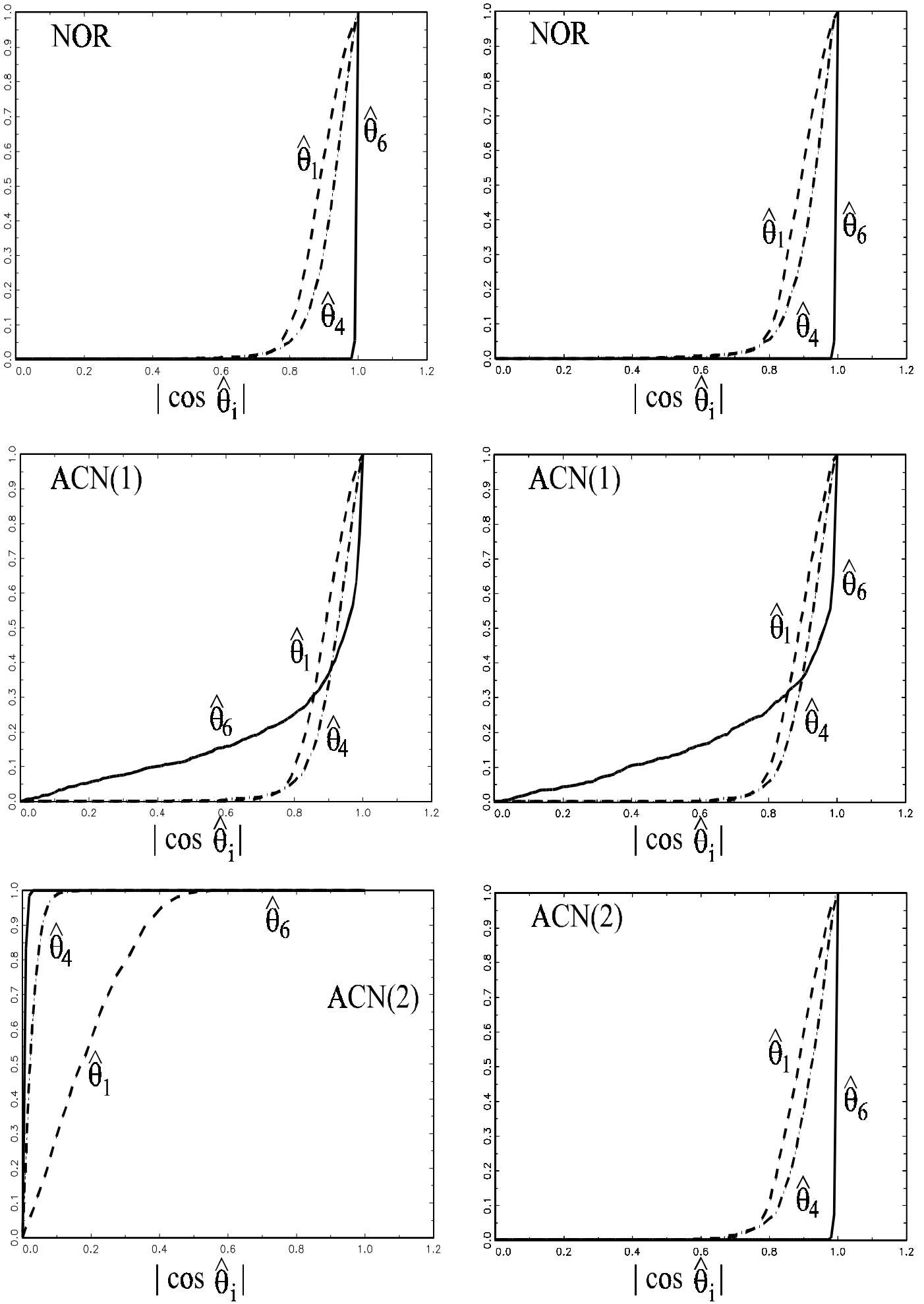

Figure 4. Simulated cumulative distribution functions for $\left|\cos \hat{\theta}_{j}\right|$, where $\hat{\theta}_{j}$ is the angle between the estimated and the population eigenvectors, for the M-estimator (first column) and the S-estimator (second column) under three different sampling schemes. 
eigenvalues and eigenvectors are defined as

$$
\operatorname{EIF}\left(x, \lambda_{\mathrm{C}, j}\right)=\operatorname{IF}\left(x, \lambda_{\mathrm{C}, j} ; \hat{F}\right)
$$

and

$$
\mathrm{EIF}\left(x, v_{\mathrm{C}, j}\right)=\operatorname{IF}\left(x, v_{\mathrm{C}, j} ; \hat{F}\right)
$$

for $j=1, \ldots, p$ and $\hat{F}=\mathrm{N}\left(t_{n}, \mathrm{C}_{n}\right)$. Similar formulas apply for the correlation case.

Diagnostics which measure the influence of the observation $x_{i}$ on the final estimates are then given by $\operatorname{EIF}\left(x_{i}, \lambda_{\mathrm{C}, j}\right)$ and $\operatorname{EIF}\left(x_{i}, v_{\mathrm{C}, j}\right)$. They may be visualized by plotting their values, or the norm of their values against the index of the observations. This is illustrated using the soil composition data set (20 observations on 4 variables) introduced by Kendall (1975), and used by all the papers mentioned before in this Section. In Figure 5, the value of $\operatorname{EIF}\left(x_{i}, \lambda_{\mathrm{R}, j}\right)$ is plotted for each observation with respect to its index, and this for every eigenvalue of the correlation matrix. The $\operatorname{EIF}\left(x_{i}, \lambda_{\mathrm{R}, j}\right)$ are computed once for the sample correlation matrix (left column), and once for the correlation estimator based on the RMCD (right column). The latter estimator was chosen because emphasis is more on exploring the data, then on inference. Moreover, the EIFs computed from S and M-estimators did not result in such a clear cut difference between the classical and robust approach.

Informal visual inspection of these plots show that observation 14 has a large influence on the second and third eigenvalues, while also observation 13 is quite influential on the first and fourth eigenvalues computed from the sample correlation matrix. This confirms the results obtained by the influence measures of Shi (1997). On the other hand, one notices that no observation has an influence which is much bigger than all the others on the eigenvalues of the RMCD correlation estimator. This is consistent with the philosophy of robustness, saying that a single observation may not influence too heavily the final estimate. Observations 13 and 14 have been downweighted by the RMCD-estimators, reason why their influence is greatly reduced.

To investigate the robustness of the index plots, contamination was introduced in the data set by changing the first and last component of the third observation (as was done in Jaupi and Saporta 1993). The "contaminated" index plots are represented on the same figures (dashed lines). First of all, notice that the EIF hardly changes for the robust RMCD estimator. The results for the classical estimator change quite a lot: one sees for example 

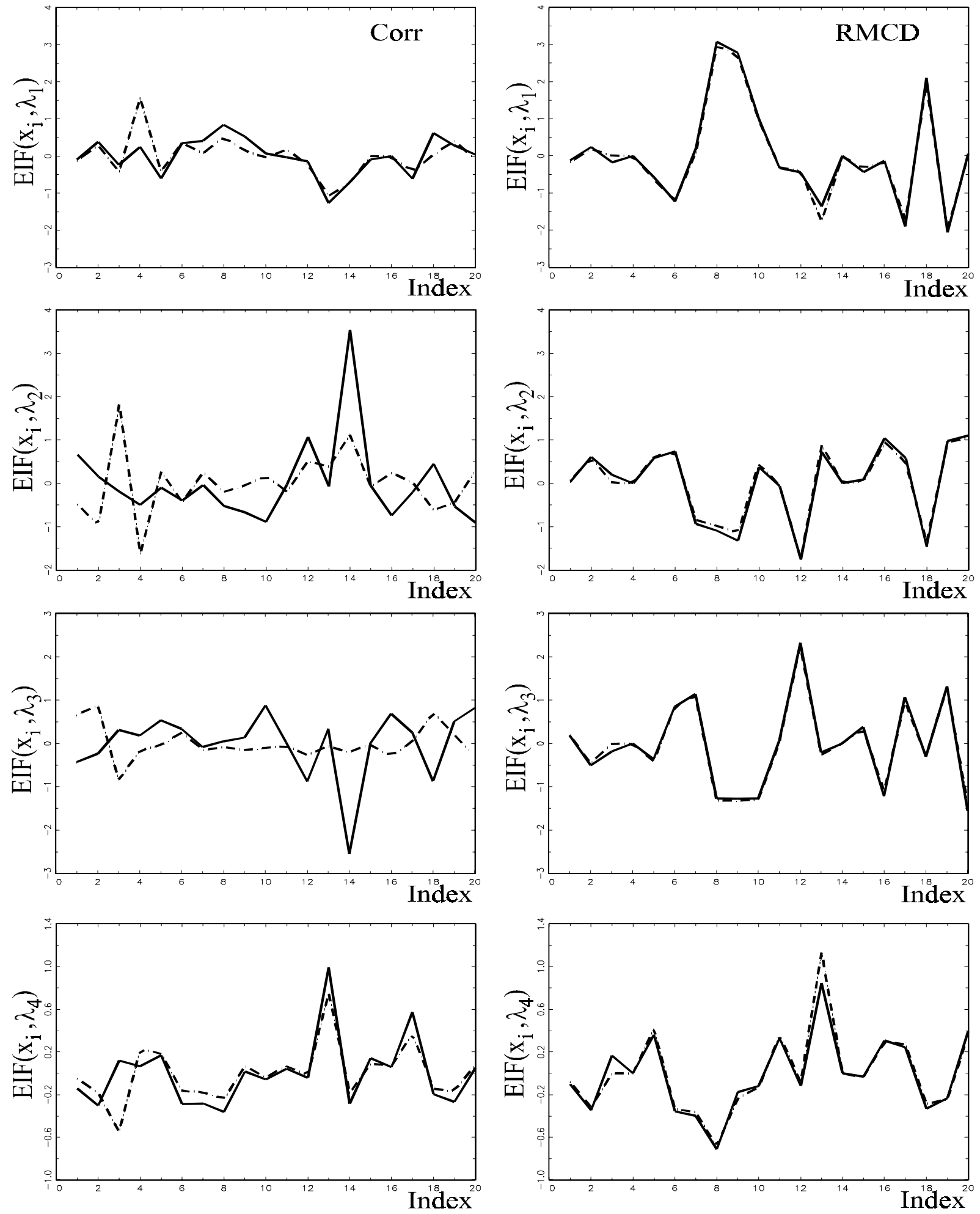

Figure 5. Empirical Influence Functions for the classical correlation matrix (left column) and for the RMCD estimator (right column) computed at the "soil"-data (solid line) and at the contaminated "soil"-data (dashed line). 
that observation 14 is not an influential point anymore. It is also interesting to notice that the contaminated observation 3 does not appear to be extremely influential on the classical estimates. Of course, 3 is neither influential on the robust estimates since it has been downweighted to zero. A conclusion is that, although EIFs can be useful to determine which points are more influential that others, they are not suitable for outlier detection.

To detect outliers, it seems to be better to use the Robust Distances, which are robustified versions of the Mahalanobis distances

$$
\mathrm{RD}_{i}=d\left(x_{i}, t_{n}, \mathrm{C}_{n}\right)=\sqrt{\left(x_{i}-t_{n}\right)^{t} \mathrm{C}_{n}^{-1}\left(x_{i}-t_{n}\right)}
$$

with $\left(t_{n}, \mathrm{C}_{n}\right)$ robust estimates of location and scatter. Observations with $\mathrm{RD}_{i}$ bigger than the critical value $\sqrt{\chi_{4,0.975}^{2}}=3.34$ can be considered as potential outliers (cfr. Rousseeuw and van Zomeren, 1990). Mahalanobis distances (obtained by using the sample mean and covariance in (5.1)) and Robust Distances based on the RMCD estimator are reported for the most interesting observations in the table below:

\begin{tabular}{|c|ccccc|ccccc|}
\cline { 2 - 10 } \multicolumn{1}{c|}{} & \multicolumn{4}{c|}{ Soil Data } & \multicolumn{4}{c|}{ Contaminated Soil Data } \\
\hline Index & 3 & 4 & 12 & 13 & 14 & 3 & 4 & 12 & 13 & 14 \\
Mahalanobis Distance & 1.21 & 3.13 & 1.96 & 2.43 & 3.04 & 4.19 & 2.80 & 1.88 & 2.35 & 2.86 \\
Robust Distance & 1.19 & 4.52 & 2.75 & 2.36 & 4.25 & 29.9 & 4.45 & 2.67 & 2.31 & 4.12 \\
\hline
\end{tabular}

Observation 4 has the largest distance for the clean data set, but is not detected as extremely influential for the eigenvalues. It was however detected by Shi (1997) as influential for the eigenvectors and it also comes up in the plot for $\lambda_{2}$ on the contaminated data. Notice that the classical Mahalanobis distance is not well suited for detecting outliers, since it is based on non robust estimators. Another tool to detect outliers is to make side by side boxplots of the scores on the robustly estimated principal components, as was suggested in (Croux and Ruiz, 1996).

\section{Conclusion}

Outlier resistant principal component estimators can be obtained by computing eigenvalues and eigenvectors of a robust estimate of the covariance or correlation matrix. Applications using M-estimators of scatter can be found in (Campbell 1980, Rivest and Plante 1988, 
Daigle and Rivest 1992). Since the breakdown point of an M-estimator decreases towards zero with the dimension, high breakdown methods seem to be preferable. The most available high breakdown scatter matrix estimators are the Minimum Volume Ellipsoid (MVE) and the Minimum Covariance Determinant estimator (Rousseeuw 1985). Simulations have been conducted to study the behavior of principal components based on the latter estimators by Naga and Antille (1990), and Todorov, Neykov and Neytchev (1992). Although they can be very useful in a first stage of a data analysis, they lack statistical efficiency. (The MVE-estimator even has a non-normal convergence, reason why it was not included in the present study.) The theoretical results and simulations in this paper favor the use of S-estimators, since they combine high efficiency with appealing robustness properties, including a smooth influence function. In an exploratory data analysis, the RMCD-approach is a valuable alternative.

General expressions for influence functions have been given, which can be used for any scatter matrix estimator possessing an influence function. From them, asymptotic variances can be computed. It was also shown how influence functions can be used as an empirical diagnostic tool.

In case that the number of variables is bigger than $n(1-\alpha)$, the $\mathrm{S}$ and RMCD method with breakpoint $\alpha \%$ are no longer applicable. (It can be seen that in this case, definition (1.1) and the definition of MCD yield an infinity of possible solutions). This is a serious drawback, since in many applications they are more variables than observations (e.g. Locantore et al (1999)). Projection based methods (Li and Chen 1985, Croux and Ruiz-Gazen 1996) may yield an outcome here.

Covariance and correlation matrices play a crucial role in many multivariate statistical techniques. Robustification of these techniques can be obtained using robust estimators for $\Sigma$ and $P$. As such, Pison et al (1999) propose a robust way to factor analysis.

\section{Appendix}

Proof of Lemma 1: For $X \sim F$, denote $F_{0}$ the distribution of $\Sigma^{-\frac{1}{2}}(X-\mu)$. Since $\mathrm{C}$ is an affine equivariant functional,

$$
\mathrm{C}(X)=\Sigma^{\frac{1}{2}} \mathrm{C}\left(\Sigma^{-\frac{1}{2}}(X-\mu)\right) \Sigma^{\frac{1}{2}}
$$


implying

$$
\operatorname{IF}(x, \mathrm{C} ; F)=\Sigma^{\frac{1}{2}} \mathrm{IF}\left(\Sigma^{-\frac{1}{2}}(x-\mu), \mathrm{C} ; F_{0}\right) \Sigma^{\frac{1}{2}} .
$$

Since $F_{0}$ is spherically symmetric, Lemma 1, page 276 in Hampel et al (1986) guarantees that two real-valued functions $\alpha_{\mathrm{C}}$ and $\beta_{\mathrm{C}}$ exist such that

$$
\operatorname{IF}\left(u, \mathrm{C} ; F_{0}\right)=\alpha_{\mathrm{C}}(\|u\|) u u^{t}-\beta_{\mathrm{C}}(\|u\|) \mathbf{I}_{p}
$$

where $\mathbf{I}_{p}$ is the $p \times p$ identity matrix and $\|$.$\| indicates the Euclidean norm. Substituting$ (7.2) in (7.1) yields

$$
\operatorname{IF}(x, \mathrm{C} ; F)=\Sigma^{\frac{1}{2}}\left\{\alpha_{\mathrm{C}}\left(\left\|\Sigma^{-\frac{1}{2}}(x-\mu)\right\|\right) \Sigma^{-\frac{1}{2}}(x-\mu)(x-\mu)^{t} \Sigma^{-\frac{1}{2}}-\beta_{\mathrm{C}}\left(\left\|\Sigma^{-\frac{1}{2}}(x-\mu)\right\|\right) \mathbf{I}_{p}\right\} \Sigma^{\frac{1}{2}} .
$$

Equation (2.3) follows now immediately by noting that $\left\|\Sigma^{-\frac{1}{2}}(x-\mu)\right\|=\left\{(x-\mu)^{t} \Sigma^{-1}(x-\right.$ $\mu)\}^{\frac{1}{2}}=d(x)$.

The proof of Theorem 1 relies on the following lemma, which mimics Lemma 2.1 of Sibson (1979) and is included for reasons of completeness:

Lemma 3. Let $S: \mathcal{F} \rightarrow S P D(p)$ be a statistical functional and $F$ a p-dimensional distribution. Suppose that $\operatorname{IF}(x, S ; F)$ exists and let $S(F)=\Xi$. Denote $v_{1}, \ldots, v_{p}$ and $\lambda_{1}, \ldots, \lambda_{p}$ the eigenvectors and eigenvalues of $\Xi$. Then the influence functions of $v_{S, j}$ and $\lambda_{S, j}(j=1, \ldots, p)$ are given by

$$
I F\left(x, \lambda_{S, j} ; F\right)=v_{j}^{t} \operatorname{IF}(x, S ; F) v_{j}
$$

and

$$
I F\left(x, v_{S, j} ; F\right)=\sum_{\substack{k=1 \\ k \neq j}}^{p} \frac{1}{\lambda_{j}-\lambda_{k}}\left(v_{k}^{t} I F(x, S ; F) v_{j}\right) v_{k} .
$$

Proof of Lemma 3: Since $\mathrm{S}\left(F_{\varepsilon}\right) v_{\mathrm{S}, j}\left(F_{\varepsilon}\right)=\lambda_{\mathrm{S}, j}\left(F_{\varepsilon}\right) v_{\mathrm{S}, j}\left(F_{\varepsilon}\right)$ for $F_{\varepsilon}=(1-\varepsilon) F+\varepsilon \Delta_{x}$, simple derivation yields

$$
\left.\frac{\partial \mathrm{S}\left(F_{\varepsilon}\right)}{\partial \varepsilon}\right|_{\varepsilon=0} v_{j}+\left.\Xi \frac{\partial v_{\mathrm{S}, j}\left(F_{\varepsilon}\right)}{\partial \varepsilon}\right|_{\varepsilon=0}=\left.\frac{\partial \lambda_{\mathrm{S}, j}\left(F_{\varepsilon}\right)}{\partial \varepsilon}\right|_{\varepsilon=0} v_{j}+\left.\lambda_{j} \frac{\partial v_{\mathrm{S}, j}\left(F_{\varepsilon}\right)}{\partial \varepsilon}\right|_{\varepsilon=0} .
$$

Rearranging the terms gives

$$
\left(\Xi-\lambda_{j} \mathbf{I}_{p}\right) \operatorname{IF}\left(x, v_{\mathrm{S}_{, j}} ; F\right)=\left(\operatorname{IF}\left(x, \lambda_{\mathrm{S}, j} ; F\right) \mathbf{I}_{p}-\mathrm{IF}(x, \mathrm{~S} ; F)\right) v_{j}
$$


which can, using $\Xi=\sum_{k=1}^{p} \lambda_{k} v_{k} v_{k}^{t}$ and $\mathbf{I}_{p}=\sum_{k=1}^{p} v_{k} v_{k}^{t}$, be written as

$$
\begin{array}{r}
\sum_{\substack{k=1 \\
k \neq j}}^{p}\left[\left(\lambda_{k}-\lambda_{j}\right) v_{k}^{t} \mathrm{IF}\left(x, v_{\mathrm{S}, j} ; F\right)\right] v_{k}=-\sum_{\substack{k=1 \\
k \neq j}}^{p}\left[v_{k}^{t} \mathrm{IF}(x, \mathrm{~S} ; F) v_{j}\right] v_{k} \\
+\left[\operatorname{IF}\left(x, \lambda_{\mathrm{S}, j} ; F\right)-v_{j}^{t} \mathrm{IF}(x, \mathrm{~S} ; F) v_{j}\right] v_{j}
\end{array}
$$

Since $v_{1}, \ldots, v_{p}$ form an orthogonal basis, (7.5) implies (7.3) and

$$
v_{k}^{t} \mathrm{IF}\left(x, v_{\mathrm{S}, j} ; F\right)=v_{k}^{t} \mathrm{IF}(x, \mathrm{~S} ; F) v_{j} /\left(\lambda_{j}-\lambda_{k}\right) .
$$

Noting that the side-condition $v_{j}^{t} v_{j}=0$ implies that $\operatorname{IF}\left(x, v_{\mathrm{S}, j} ; F\right)$ has no component in the direction of $v_{j},(7.4)$ follows.

Proof of Theorem 1: From Lemma 1, there exist two real-valued functions $\alpha_{\mathrm{C}}$ and $\beta_{\mathrm{C}}$ such that

$$
v_{k}^{t} \operatorname{IF}(x, \mathrm{C} ; F) v_{j}=\alpha_{\mathrm{C}}(d(x))\left(v_{k}^{t}(x-\mu)\right)\left((x-\mu)^{t} v_{j}\right)-\beta_{\mathrm{C}}(d(x)) v_{k}^{t} \Sigma v_{j}
$$

Lemma 3 combined with (7.6) and the equality $v_{k}^{t} \Sigma v_{j}=\lambda_{j} \delta_{j k}$ results in

$$
\operatorname{IF}\left(x, \lambda_{\mathrm{C}, j} ; F\right)=v_{j}^{t} \operatorname{IF}(x, \mathrm{C} ; F) v_{j}=\alpha_{\mathrm{C}}(d(x))\left(v_{j}^{t}(x-\mu)\right)^{2}-\beta_{\mathrm{C}}(d(x)) \lambda_{j}
$$

and

$$
\operatorname{IF}\left(x, v_{\mathrm{C}, j} ; F\right)=\sum_{\substack{k=1 \\ k \neq j}}^{p} \frac{1}{\lambda_{j}-\lambda_{k}} \alpha_{\mathrm{C}}(d(x))\left(v_{k}^{t}(x-\mu)\right)\left(v_{j}^{t}(x-\mu)\right)^{t} v_{k} .
$$

Replacing $v_{j}^{t}(x-\mu)$ by the $z$-score $z_{j}$ yields the stated expressions.

Proof of Corollary 1: Using (2.6) and Theorem 1, the asymptotic variance of $\lambda_{C_{, j}}$ can be computed as:

$$
\operatorname{ASV}\left(\lambda_{\mathrm{C}, j}, F\right)=E_{F}\left[\operatorname{IF}\left(x, \lambda_{\mathrm{C}, j} ; F\right)^{2}\right]=E_{F}\left[\left(\alpha_{\mathrm{C}}(d(x)) z_{j}^{2}-\beta_{\mathrm{C}}(d(x)) \lambda_{j}\right)^{2}\right]
$$

where $z_{j}=v_{j}^{t}(x-\mu)$.

With $u_{j}=z_{j} / \sqrt{\lambda_{j}}$, one has that $u=\left(u_{1}, \ldots, u_{p}\right)^{t} \sim F_{0}$. Moreover, $d^{2}(x)=\sum_{j=1}^{p} u_{j}^{2}$ and Lemma 1 imply

$$
\begin{aligned}
\operatorname{ASV}\left(\lambda_{\mathrm{C}, j}, F\right) & =E_{F_{0}}\left[\left(\alpha_{\mathrm{C}}(\|u\|) \lambda_{j} u_{j}^{2}-\beta_{\mathrm{C}}(\|u\|) \lambda_{j}\right)^{2}\right] \\
& =\lambda_{j}^{2} E_{F_{0}}\left[\operatorname{IF}\left(u, \mathrm{C}_{j j} ; F_{0}\right)^{2}\right]=\lambda_{j}^{2} \operatorname{ASV}\left(\mathrm{C}_{11}, F_{0}\right)
\end{aligned}
$$


as $\mathrm{ASV}\left(\mathrm{C}_{j j}, F_{0}\right)=\mathrm{ASV}\left(\mathrm{C}_{11} ; F_{0}\right)$ by symmetry. For the eigenvector estimator, the asymptotic variance is given by

$$
\begin{aligned}
\operatorname{ASV}\left(v_{\mathrm{C}, j}, F\right) & =E_{F}\left[\operatorname{IF}\left(x, v_{\mathrm{C}, j} ; F\right) \operatorname{IF}\left(x, v_{\mathrm{C}, j} ; F\right)^{t}\right] \\
& =\sum_{\substack{k=1 \\
k \neq j}}^{p} \sum_{\substack{l=1 \\
l \neq j}}^{p} \frac{1}{\lambda_{j}-\lambda_{k}} \frac{1}{\lambda_{j}-\lambda_{l}} E_{F}\left[\alpha_{\mathrm{C}}(d(x))^{2} z_{k} z_{l} z_{j}^{2}\right] v_{k} v_{l}^{t} .
\end{aligned}
$$

Using again the transformation $z_{j} / \sqrt{\lambda_{j}}=u_{j}$ to compute the expectation in (7.7) leads to

$$
E_{F}\left[\alpha_{\mathrm{C}}(d(x))^{2} z_{k} z_{l} z_{j}^{2}\right]=\sqrt{\lambda_{k}} \sqrt{\lambda_{l}} \lambda_{j} E_{F_{0}}\left[\alpha_{\mathrm{C}}(\|u\|)^{2} u_{k} u_{l} u_{j}^{2}\right]=\lambda_{k} \lambda_{j} E_{F_{0}}\left[\alpha_{\mathrm{C}}(\|u\|)^{2} u_{k}^{2} u_{j}^{2}\right] \delta_{k l} .
$$

Substituting (7.8) in (7.7) and using Lemma 1 yields

$$
\begin{aligned}
\operatorname{ASV}\left(v_{\mathrm{C}, j}, F\right) & =\sum_{\substack{k=1 \\
k \neq j}}^{p} \frac{\lambda_{j} \lambda_{k}}{\left(\lambda_{j}-\lambda_{k}\right)^{2}} E_{F_{0}}\left[\operatorname{IF}\left(u, \mathrm{C}_{j k} ; F_{0}\right)^{2}\right] v_{k} v_{k}^{t} \\
& =\operatorname{ASV}\left(\mathrm{C}_{12}, F_{0}\right) \sum_{\substack{k=1 \\
k \neq j}}^{p} \frac{\lambda_{j} \lambda_{k}}{\left(\lambda_{j}-\lambda_{k}\right)^{2}} v_{k} v_{k}^{t}
\end{aligned}
$$

since $\operatorname{ASV}\left(\mathrm{C}_{j k}, F_{0}\right)=\operatorname{ASV}\left(\mathrm{C}_{12}, F_{0}\right)$ by symmetry.

Proof of Lemma 2: Since $\mathrm{R}\left(F_{\varepsilon}\right)=\mathrm{C}_{D}^{-\frac{1}{2}}\left(F_{\varepsilon}\right) \mathrm{C}\left(F_{\varepsilon}\right) \mathrm{C}_{D}^{-\frac{1}{2}}\left(F_{\varepsilon}\right)$ with $F_{\varepsilon}=(1-\varepsilon) F+\varepsilon \Delta_{x}$, $\mathrm{C}(F)=\Sigma$ and $\mathrm{C}_{D}(F)=\Sigma_{D}$, derivation yields

$$
\operatorname{IF}(x, \mathrm{R} ; F)=-\frac{1}{2}\left\{\operatorname{IF}\left(x, \mathrm{C}_{D} ; F\right) \Sigma_{D}^{-1} R+R \Sigma_{D}^{-1} \operatorname{IF}\left(x, \mathrm{C}_{D} ; F\right)\right\}+\Sigma_{D}^{-\frac{1}{2}} \mathrm{IF}(x, \mathrm{C} ; F) \Sigma_{D}^{-\frac{1}{2}} .
$$

¿From Lemma 1 , there exist two functions $\alpha_{\mathrm{C}}$ and $\beta_{\mathrm{C}}$ such that

$$
\operatorname{IF}\left(x, \mathrm{C}_{D} ; F\right)=\alpha_{\mathrm{C}}(d(x)) D_{x}-\beta_{\mathrm{C}}(d(x)) \Sigma_{D}
$$

with $D_{x}=\operatorname{diag}\left((x-\mu)(x-\mu)^{t}\right)$. Inserting (2.3) and (7.10) in (7.9) yields

$$
\operatorname{IF}(x, R ; F)=-\frac{1}{2} \alpha_{\mathrm{C}}(d(x))\left(D_{x} \Sigma_{D}^{-1} R+R \Sigma_{D}^{-1} D_{x}\right)+\alpha_{\mathrm{C}}(d(x))\left(\Sigma_{D}^{-\frac{1}{2}}(x-\mu)\right)\left(\Sigma_{D}^{-\frac{1}{2}}(x-\mu)\right)^{t} .
$$

Putting $\tilde{x}=\Sigma_{D}^{-\frac{1}{2}}(x-\mu)$ and $D_{x} \Sigma_{D}^{-1}=\Sigma_{D}^{-1} D_{x}=D_{\tilde{x}}$ completes the proof.

Proof of Theorem 2: From Lemma 2, it follows that

$$
v_{j}^{t} \operatorname{IF}(x, R ; F) v_{k}=\alpha_{\mathrm{C}}(d(x))\left\{\left(v_{j}^{t} \tilde{x}\right)\left(v_{k}^{t} \tilde{x}\right)^{t}-\left(\frac{v_{j}^{t} D_{\tilde{x}} R v_{k}+\left(R v_{j}\right)^{t} D_{\tilde{x}} v_{k}}{2}\right)\right\} .
$$


With $R v_{k}=\lambda_{k} v_{k}$ and $R v_{j}=\lambda_{j} v_{j}$, Lemma 3 gives

$$
\operatorname{IF}\left(x, \lambda_{\mathrm{R}, j} ; F\right)=v_{j}^{t} \operatorname{IF}(x, \mathrm{R} ; F) v_{j}=\alpha_{\mathrm{C}}(d(x))\left(\tilde{z}_{j}^{2}-\lambda_{j} v_{j}^{t} D_{\tilde{x}} v_{j}\right)
$$

and

$$
\operatorname{IF}\left(x, v_{\mathrm{R}, j} ; F\right)=\alpha_{\mathrm{C}}(d(x)) \sum_{\substack{k=1 \\ k \neq j}}^{p}\left(\tilde{z}_{k} \tilde{z}_{j}-\frac{\lambda_{k}+\lambda_{j}}{2} v_{j}^{t} D_{\tilde{x}} v_{k}\right) \frac{v_{k}}{\lambda_{j}-\lambda_{k}} .
$$

\section{References}

Anderson, T.W. (1963), "Asymptotic Theory for Principal Components Analysis", The Annals of Mathematical Statistics, 34, 122-148.

Boente, G. (1987), “Asymptotic Theory for Robust principal Components", Journal of Multivariate Analysis, 21, 67-78.

Campbell, N.A. (1980), "Robust Procedures in Multivariate Analysis: Robust Covariance Estimation", Applied Statistics, 29, 5-14.

Critchley, F. (1985), "Influence in Principal Component Analysis", Biometrika, 72, 627-636.

Croux, C., and Haesbroeck, G. (1998), "Influence Function and Efficiency of the Minimum Covariance Determinant Scatter Matrix Estimator", to appear in The Journal of Multivariate Analysis.

Croux, C. and Ruiz-Gazen, A. (1996), "A Fast Algorithm for Robust Principal Components based on Projection Pursuit," in Compstat: Proceedings in Computational Statistics, ed.A. Prat, Heidelberg: Physica-Verlag. 211-217.

Daigle, G., and Rivest, L-P. (1992), "A Robust Biplot", The Canadian Journal of Statistics, $120,241-255$.

Davies, P.L. (1987), "Asymptotic Behavior of S-Estimators of Multivariate Location Parameters and Dispersion Matrices", The Annals of Statistics, 15, 1269-1292.

Devlin, S.J., Gnanadesikan, R., and Kettenring, J.R. (1975), "Robust Estimation and Outlier Detection with Correlation Coefficients", Biometrika, 62, 531-545.

Devlin, S.J., Gnanadesikan, R., and Kettenring, J.R. (1981), "Robust Estimation of Dispersion Matrices and Principal Components", Journal of the American Statistical Association, 76, 354-362. 
Hampel, F.R., Ronchetti, E.M., Rousseeuw, P.J., and Stahel, W.A. (1996), Robust Statistics: The Approach based on Influence Functions, New York: Wiley.

Huber, P.J. (1981), Robust Statistics, New York: Wiley.

Jackson, J.E. (1991), A User's Guide to Principal Components, New York: Wiley.

Jaupi, L., and Saporta, G. (1993), "Using the Influence Function in Robust Principal Components Analysis", New Directions in Statistical Data Analysis and Robustness, eds. S. Morgenthaler, E. Ronchetti, and W.A. Stahel, Basel: Birkhäuser, 147-156.

Joliffe, I.T. (1986), Principal Component Analysis, New York: Springer-Verlag.

Kendall, M. (1975), Multivariate Analysis, Charles Griffin \& Company LTD.

Li, G. and Chen, Z (1985), "Projection-Pursuit Approach to Robust Dispersion Matrices and Principal Components : Primary Theory and Monte Carlo", Journal of the American Statistical Association, 80, 759-766.

Locantore, N., Marron, J.S., Simpson, D.G., Tripoli, N., Zhang, J.T., and Cohen, K.L. (1999), "Robust Principal Components for Functional Data," to appear in Test.

Lopuhaä, H.P. (1989), "On the Relation Between S-estimators and M-estimators of Multivariate Location and Covariance", The Annals of Statistics, 17, 1662-1683.

Lopuhaä, H.P. (1997), "Asymptotics of Reweighted Estimators of Multivariate Location and Scatter", preprint TU Delft.

Maronna, R.A. (1976), "Robust M-estimators of Multivariate Location and Scatter", The Annals of Statistics, 4, 51-67.

Maronna, R.A., and Yohai (1998), "Robust Estimation of Multivariate Location and Scatter", in Encyclopedia of Statistical Sciences Update Volume 2, eds. S. Kotz, C. Read, and D. Banks, New York: Wiley, 589-596.

Naga, R., and Antille, G. (1990), "Stability of Robust and Non-Robust Principal Component Analysis," Computational Statistics \& Data Analysis, 10, 169-174.

Pison, G., Rousseeuw, P.J., Filzmoser, P. and Croux, C. (1999), "Factor Analysis in a Robust Way", preprint University of Antwerp.

Rivest, L-P., and Plante, N. (1988), "L'Analyse en Composantes Principales Robuste", Revue Statistique Appliquée, XXXVI (1), 55-66. 
Rousseeuw, P.J. (1985), "Multivariate Estimation with High Breakdown Point", in Mathematical Statistics and Applications, Vol. B, eds. W. Grossmann, G. Pflug, I. Vincze, and W. Wertz, Dordrecht: Reidel, 283-297.

Rousseeuw, P.J., and Leroy, A.M. (1987), Robust Regression and Outlier Detection, New York: Wiley.

Rousseeuw, P.J., and Van Driessen, K. (1997), "A Fast Algorithm for the Minimum Covariance Determinant Estimator", preprint University of Antwerp.

Rousseeuw, P.J., and van Zomeren, B.C. (1990), "Unmasking Multivariate Outliers and Leverage Points", Journal of the American Statistical Association, 85, 633-639.

Ruppert, D. (1992), "Computing S-Estimators for Regression and Multivariate Location/Dispersion", Journal of Computational and Graphical Statistics, 1, 253-270.

Shi, L. (1997), "Local Influence in Principal Components Analysis", Biometrika, 84, 175-186.

Sibson, R. (1979), "Studies in the Robustness of Multidimensional Scaling: Perturbational Analysis of Classical Scaling", Journal of the Royal Statistical Society B, 41, 217-229.

Tanaka, Y. (1988), "Sensitivity Analysis in PCA: Influence on the Subspace Spanned by Principal Components", Communications in Statistics: Theory and Methods, 17, 31573175 .

Todorov, V.K., Neykov N., and Neytchev, P.N. (1992), "Stability of (High Breakdown Point) Robust Principal Components Analysis," in COMPSTAT 1994, Short Communications in Computational Statistics, eds. R. Dutter and W. Grossmann, 90-92. 